Article

\title{
Bayesian Model for Matching the Radiometric Measurements of Aerospace and Field Ocean Color Sensors
}

\section{Mhd. Suhyb Salama ${ }^{\star}$ and Zhongbo Su}

Faculty of Geo-Information Science and Earth Observation (ITC), University of Twente, Hengelosestraat 99, 7500 AA Enschede, The Netherlands; E-Mail: b_su@itc.nl

* Author to whom correspondence should be addressed; E-Mail: salama@itc.nl; Tel.: +31 0534874288 ; Fax: +310534874336.

Received: 11 June 2010; in revised form: 16 July 2010 / Accepted: 10 August 2010 /

Published: 11 August 2010

\begin{abstract}
A Bayesian model is developed to match aerospace ocean color observation to field measurements and derive the spatial variability of match-up sites. The performance of the model is tested against populations of synthesized spectra and full and reduced resolutions of MERIS data. The model derived the scale difference between synthesized satellite pixel and point measurements with $\mathrm{R}^{2}>0.88$ and relative error $<21 \%$ in the spectral range from $400 \mathrm{~nm}$ to $695 \mathrm{~nm}$. The sub-pixel variabilities of reduced resolution MERIS image are derived with less than $12 \%$ of relative errors in heterogeneous region. The method is generic and applicable to different sensors.
\end{abstract}

Keywords: Bayesian; maximum entropy; match-up; ocean color; MERIS

\section{Introduction}

Consistent and accurate matching of aerospace observation data to field measurements are necessary calibration and validation steps towards creating reliable products of inherent optical properties (IOPs). The scientific procedure to match satellite observations to field measurements can generally be divided into three steps: $i$ - measure remote sensing reflectance above the target in an area with homogenous optical properties; $i i$ - re-sample the pixels of satellite data that surround the field site; $i i i$ - match the values obtained from step $i$ to those computed from step $i$. Accurate estimation of the sub-scale variability of 
the match-up site and its inclusion in the matching method is the most suitable approach to calibrate earth observation retrieval algorithms and validate their products.

Matching procedures for ocean color sensors were addressed by many researchers. For instance, Harding et al. [1] selected field measurement sites in homogenous areas and Bailey and Werdell, [2] averaged a number of spatially-homogeneous pixels surrounding the match-up site. Although, aggregating ocean color pixels was found to be suitable for open ocean $[3,4]$, it lowers the percentage of usable match-up points considerably and should be avoided in coastal waters [5]. Any direct matching in coastal turbid waters may result in large discrepancy [6,7]. Hyde et al. [8] recognized that the mismatch between field measurement and SeaWiFS products of chlorophyll-a is partially due to difference in the sampling scales and therefore introduced a correction factor to overcome this scale mismatch.

In this paper we introduce a complete scheme to quantify the scale difference between a satellite pixel and a point (field) measurement. We used Bayesian inference method [9] to estimate the probability distribution function (PDF) of the match-up pixel using the deviations between field and satellite measurements. We will discuss the methodology and performance of the model as applied to radiative transfer simulations [10, IOCCG data set] and MERIS images at full and reduced resolutions acquired over the Dutch Bight.

\section{Method}

\subsection{Ocean color model}

In this study we will use the model of Gordon et al. [11] to relate the observed remote sensing reflectance that is leaving the water body $R s_{w}(\lambda)$ to the water biophysical properties:

$$
R s_{w}(\lambda)=\frac{t}{n_{w}^{2}} \sum_{i=1}^{2} g_{i}\left(\frac{b_{b}(\lambda)}{b_{b}(\lambda)+a(\lambda)}\right)^{i}
$$

where $g_{1}, g_{2}$ are subsurface expansion coefficients due to internal refraction, reflection and sun zenith; $t$ and $n_{w}$ are the sea air transmission and water index of refraction, respectively. Their values are taken to be: $g_{1}=0.0949, g_{2}=0.0794, t=0.95, n_{w}=1.34$. The quantities $b_{b}(\lambda)$ and $a(\lambda)$ are the bulk backscattering and absorption coefficients of the water column. Case II water is considered with five independently varying constituents, namely: water molecules, chlorophyll-a (Chl-a), detritus, dissolved organic matter and suspended particulate matter (SPM). The absorption $a(\lambda)$ and backscattering $b_{b}(\lambda)$ coefficients are parameterized as reported in Salama et al.[12,13] and summarized in Appendix 1. We will consider three main variables as derived from (1) using the method of Salama et al. [12]. These variables are: (i) - the absorption coefficient of Chl-a at $440 \mathrm{~nm}, a_{p h y}(440)$; (ii) - the combined absorptions of detritus and dissolved organic matter at $440 \mathrm{~nm}, a_{d g}(440)$; (iii) - the scattering coefficient of SPM at $550 \mathrm{~nm}, b_{\text {spm }}(550)$. The derived variables are called the set of IOPs and denoted in a vector notation iop. 


\subsection{Bayesian inference}

Our basic assumption is that each pixel in aerospace ocean color data represents the mean of an unknown theoretical probability distribution function (PDF) over a pixel area. In this respect, any field measured radiance is a sample drawn from this PDF [14]. Our objective is to estimate the standard deviation of this PDF which represents the scale difference between pixel and point measurement. This standard deviation is also equal to the sub-scale variability of the satellite pixel. We will use the words (sub-scale variability) to represents the scale-difference and quantify the standard deviation as its measure.

The radiometric mismatch, between aerospace and field sensors, is attributed to the scale difference and is represented as an upper and lower bounds with a 1- $\alpha$ of confidence using the method of Bates and Watts [15]:

$$
R s_{ \pm}=R s(\lambda) \pm \sigma\left\|\frac{\Delta R s(\lambda)}{\Delta \mathbf{i o p}} \mathbf{R}^{-1}\right\| t(N-m, \alpha / 2)
$$

where, $R s_{ \pm}$is the upper "+" and lower "-" bounds of $R s(\lambda) ; R s(\lambda)$ is the observed radiometric quantity; $\sigma$ is the standard deviation of the radiometric difference between field and aerospace measurements; $t(N-m, \alpha / 2)$ is the upper quantile for a Student's $t$ distribution with $N-m$ degrees of freedom. $N$ is the number of bands and $m$ is the number of unknowns. $\mathbf{R}$ is the upper triangle matrix of QR decomposition of the jacobian matrix. The derivative term in (2), can be approximated as being the gradient of (1) with respect to the derived IOPs and is computed for model-best-fit to the observation. This approximation is derived in Appendix 2.

The plausible range of the IOPs can be estimated from the upper and lower radiometric bounds by simply inverting (1) for the upper and lower radiometric bounds. A first estimate of the IOPs standard deviation is then derived form their plausible range using the method of Salama and Stein [9]. This method is summarized in Appendix 3. Campbell [16] showed that marine biophysical quantities are most likely log-normally distributed. We, therefore, use a log-normal distribution to generates random IOPs values within their plausible range using the estimated variance. We call these generated IOPs values a prior PDF of IOPs. The posterior probability of IOPs is then derived by maximizing the cross entropy $\mathrm{H}$ between prior and posterior information [17,18]:

$$
\mathrm{H}=\sum_{1}^{N} \mathrm{P}(\mathbf{i o p} \mid \ell) \cdot \log \frac{\mathrm{P}(\mathbf{i o p} \mid \ell)}{\mathrm{P}(\mathbf{i o p})}
$$

where $\mathrm{P}(\mathbf{i o p})$ is the prior probability and $\mathrm{P}(\operatorname{iop} \mid \ell)$ is the posterior probability of iop given the IOPs range $\ell$. The empirical PDF of spectra is estimated from permutated values of the derived posterior PDF of IOPs. The importance of permutation is to simulate the ambiguity of remote sensing reflectance [19] with respect to different sets of IOPs, i.e., similar spectra corresponding to different sets of IOPs.

\subsection{Algorithm}

The above-mentioned theoretical derivation of the Bayesian inference are summarize in the following algorithm: 
1. use (2) to estimate the upper and lower bounds of spectra;

2. derive the IOPs ranges from these spectra by inverting (1);

3. use the method of section 3 to estimate the standard deviation of IOPs from their range;

4. generate the prior PDF of IOPs using log-normal distribution;

5. initiate the posterior using log-normal distribution;

6. maximize (3) to obtain an new estimate of the posterior;

7. update the posterior;

8. iterate between steps (5) and (6) till convergence;

9. permutate the resulting PDF of IOPs;

10. forward the sets of IOPs using (1) to obtain the empirical PDF of spectra.

\section{Results}

\subsection{Simulated data set}

The proposed model was applied on [10, IOCCG data set] of radiative transfer simulations at $30^{\circ}$ sun zenith for synthesized sets of IOPs. The spectral arithmetic mean of this data set was computed to represent the observed aerospace spectrum. Spectra that corresponded to quantiles $0.05,0.25,0.5,0.75$, 0.9 and 0.95 were chosen to represent probable in-situ observations. The empirical distributions of the underlying population were derived and plotted in Figure 1.

Note that reflectance values are also log-normally distributed. Figure 1 shows that the distribution resulting from the 0.5 quantile has the largest kurtosis and the smallest deviation from the theoretical mean. We performed modified Ansari-Bradley test [20] at 5\% significant level on the PDFs in Figure 1. This technique is a non-parametric two-samples test on dispersions of the theoretical and empirical PDFs. The results of the Ansari-Bradley test are shown in Table 1 as probabilities of the empirical PDF having the same dispersion as the theoretical PDF for radiometric and geo-biophysical quantities.

The Ansari-Bradley test shows that wavelength $495 \mathrm{~nm}$ is more probable to produce the sought variability regardless of the position of in-situ measurements in the theoretical PDF. Our model is more likely to derive the variability of all IOPs from the 0.95 quantile. The probability of deriving the variability of $a_{d g}(440)$ and $b_{s p m}(550)$ is higher than deriving the variability of $a_{p h y}(440)$. Figure 2 shows the relationships between the known and derived standard deviation with root-mean of squared errors (RMSE) and $\mathrm{R}^{2}$ values derived from model-II regression [21] of log-transformed radiometric data. The variability obtained from all quantiles have very close values to the theoretical variability with $\mathrm{R}^{2}$ larger than 0.88. The RMSE values almost increase towards the median and start decreasing to reach a minimum value of 0.17 for 0.95 quantile. Close quantiles to the median (0.5) reproduce PDF with small dispersions. Table 2 shows the relative errors between known and derived (log) standard deviation. For all combinations of quantiles and wavelengths, the retrieved variabilities of reflectance were within $21 \%$ 
of known values. The root-mean of squared relative errors (RMS-RE) between known $c$ and derived $x$ values was computed for all wavelengths as shown in the last row of Table 2:

$$
\mathrm{RMS}-\mathrm{RE}=\sqrt{\frac{1}{n} \sum_{i=1}^{n}\left(100 \frac{x_{i}-c}{c}\right)^{2}}
$$

where $n$ in this case is the number of bands. The best results are obtained from field spectrum corresponding to the 0.95 quantile and RMS-RE values have the same trends as RMSE values shown in Figure 2, i.e., increases towards the median from both sides.

Figure 1. Empirical PDFs generated from field spectra corresponding to predefined quantiles: 0.05 "doted line", 0.25 "dashed line", 0.5 "full line", 0.75 "plus", 0.9 "square", 0.95 "circles" for six wavelengths. The theoretical PDF is illustrate as gray area.
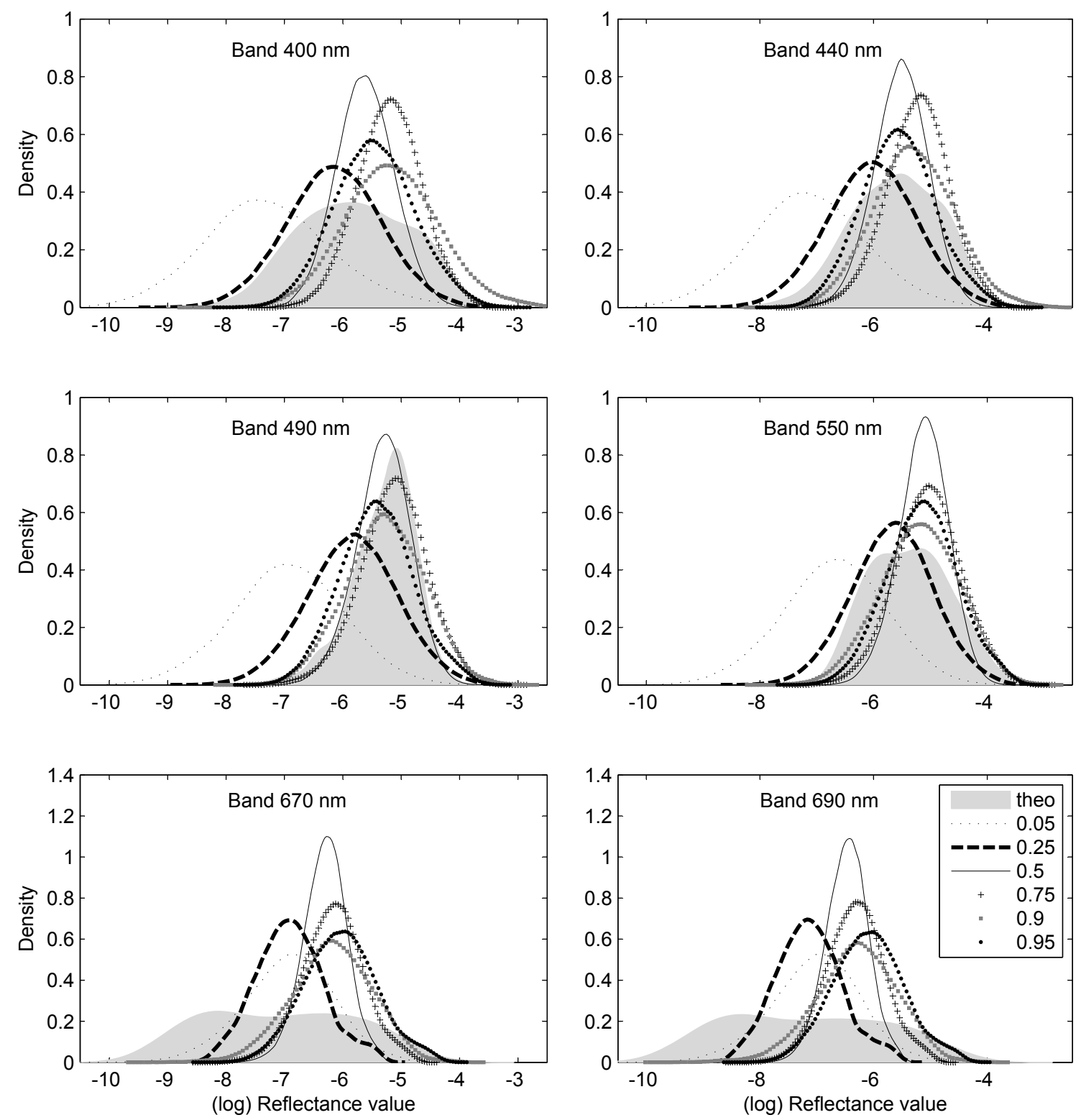
Table 1. Probability of empirical PDF having the same variability of the theoretical PDF.

\begin{tabular}{lllllll}
\hline & \multicolumn{6}{c}{ Quantile values } \\
\cline { 2 - 7 } band nm/IOPs & 0.05 & 0.25 & 0.5 & 0.75 & 0.9 & 0.95 \\
\hline 440 & 0 & 0.26 & 0 & 0 & 0.31 & 0 \\
495 & 0.59 & 0.19 & 0.17 & 0.2 & 0.11 & 0.13 \\
550 & 0 & 0.01 & 0 & 0.03 & 0.06 & 0.57 \\
\hline$a_{p h y}(440)$ & 0 & 0 & 0.09 & 0.17 & 0 & 0.18 \\
$a_{d g}(440)$ & 0 & 0.93 & 0.09 & 0.06 & 0.37 & 0.58 \\
$b_{s p m}(550)$ & 0.33 & 0.21 & 0.34 & 0.51 & 0.05 & 0.3 \\
\hline
\end{tabular}

Figure 2. Known versus derived variability corresponding to predefined quantiles on a log-scale of radiometric data. The 1:1 line is also shown.
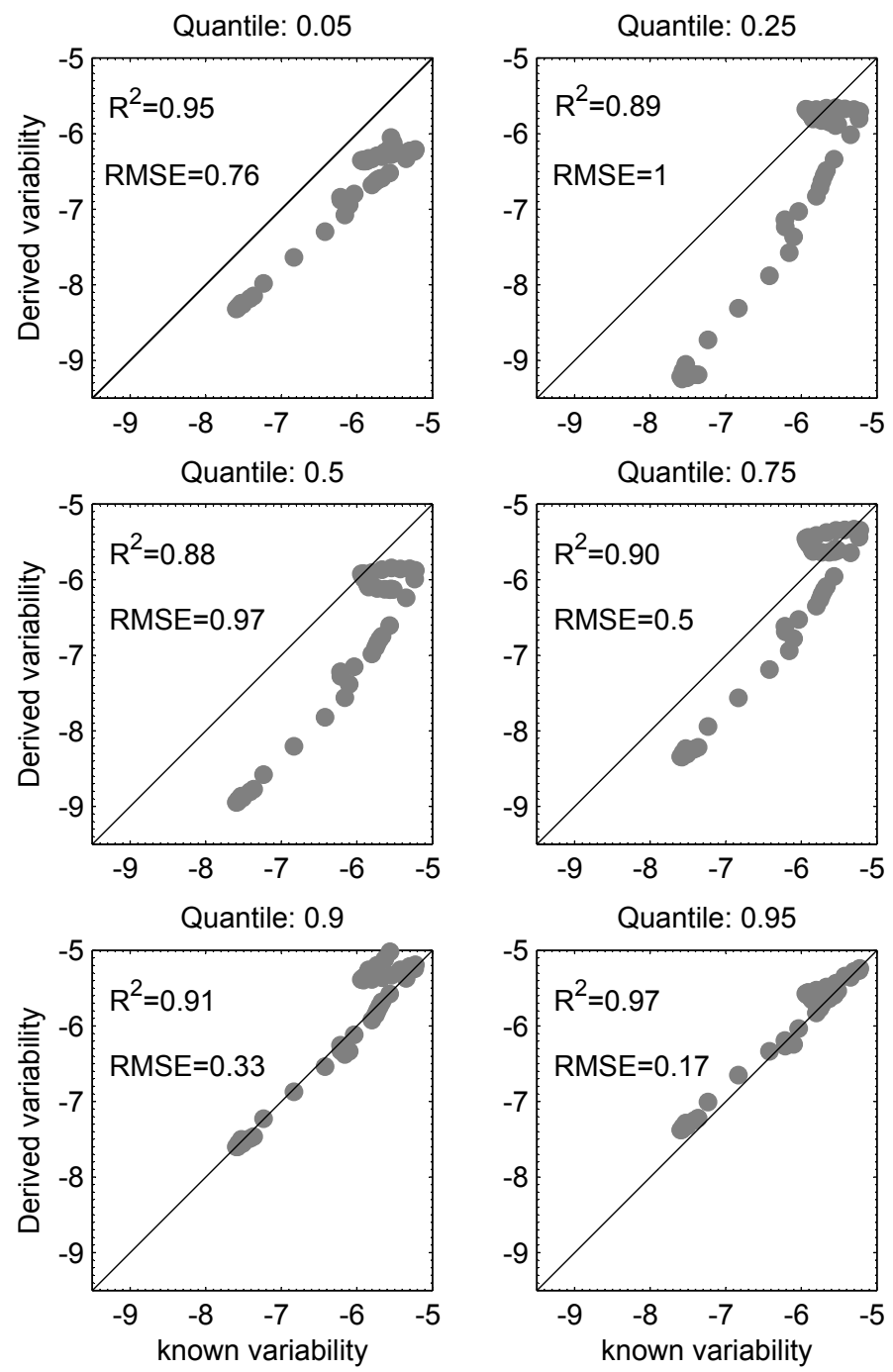
Table 2. Relative errors (\%) in derived $\log \sigma$ values of reflectance estimated from different quantile values. Minus values are underestimated. RMS-RE is the root-mean of squared relative error for all bands.

\begin{tabular}{lllllll}
\hline & \multicolumn{6}{c}{ Quantile values } \\
\cline { 2 - 7 } band nm & 0.05 & 0.25 & 0.5 & 0.75 & 0.9 & 0.95 \\
\hline 400 & 9.08 & 6.24 & 10.39 & 0.77 & -13.12 & -1.29 \\
440 & 9.78 & 1.67 & 6.71 & -1.65 & -9.29 & -1.16 \\
495 & 7.29 & -3.91 & 0.45 & -7.32 & -9.34 & -5.74 \\
550 & 15.35 & 4.57 & 8 & -1.52 & -3.06 & -1.53 \\
675 & 10.05 & 14.81 & 16.11 & 6.4 & 0.61 & -0.4 \\
695 & 13.77 & 20.7 & 20.99 & 11.12 & 3.84 & 2.32 \\
\hline RMS-RE & 12.5 & 14.6 & 14.8 & 7.6 & 5.7 & 2.7 \\
\hline
\end{tabular}

\subsection{Model stability to atmospheric and random noise}

The stability of the model to uncertainties in atmospheric correction and sensor noise was tested by perturbing the mean, i.e., aerospace spectrum, with random normal fluctuations. The random perturbations were assumed to be wavelength dependent with zero mean and standard deviation calculated from the theoretical PDF. This implies that the residuals between the aerospace and field spectra are now due to three components, namely: errors originated from atmospheric correction, sensor noise and the spatial scale difference. The added fluctuations are within $\pm 50 \%$ of the actual values as shown in appendix Figure 3. RMS-RE is computed between the log of known and derived estimates using (4) with $n$ being the number of fluctuations. Figure 4 shows that the RMS-RE values are less than $15 \%$ for derived variability values from the 0.05 and 0.95 quantiles. Derived values from 0.95 quantiles were more accurate than those derived using the 0.05 quantiles for wavelengths $>500 \mathrm{~nm}$.

\subsection{Ocean color radiometric products}

We used full (FR) and reduced (RR) resolutions of MERIS images acquired over the North Sea [22]. Atmospheric path correction was then performed using radiative transfer computation [23] and the method of Salama and Shen [24]. For this study, a region of the Dutch coastal waters was selected with relatively high optical variability. The sub-pixel variability of the reduced resolution image was derived for selected quantiles from the full resolution image. Figure 5 shows the relative errors of the derived variability for band 6 of MERIS centered at $620 \mathrm{~nm}$. The derived (log) standard deviation values were within $10 \%$ of relative errors in spatially variable areas and up to $40 \%$ in spatially homogenous waters. The mean and standard deviation of RMS-RE values of the images in Figure 5 (c-h) were between $7 \%$ and $8 \%$ and $7.3 \%$ and $7.5 \%$ respectively. 
Figure 3. Relative values of fluctuations added to the aerospace mean for six wavelengths.
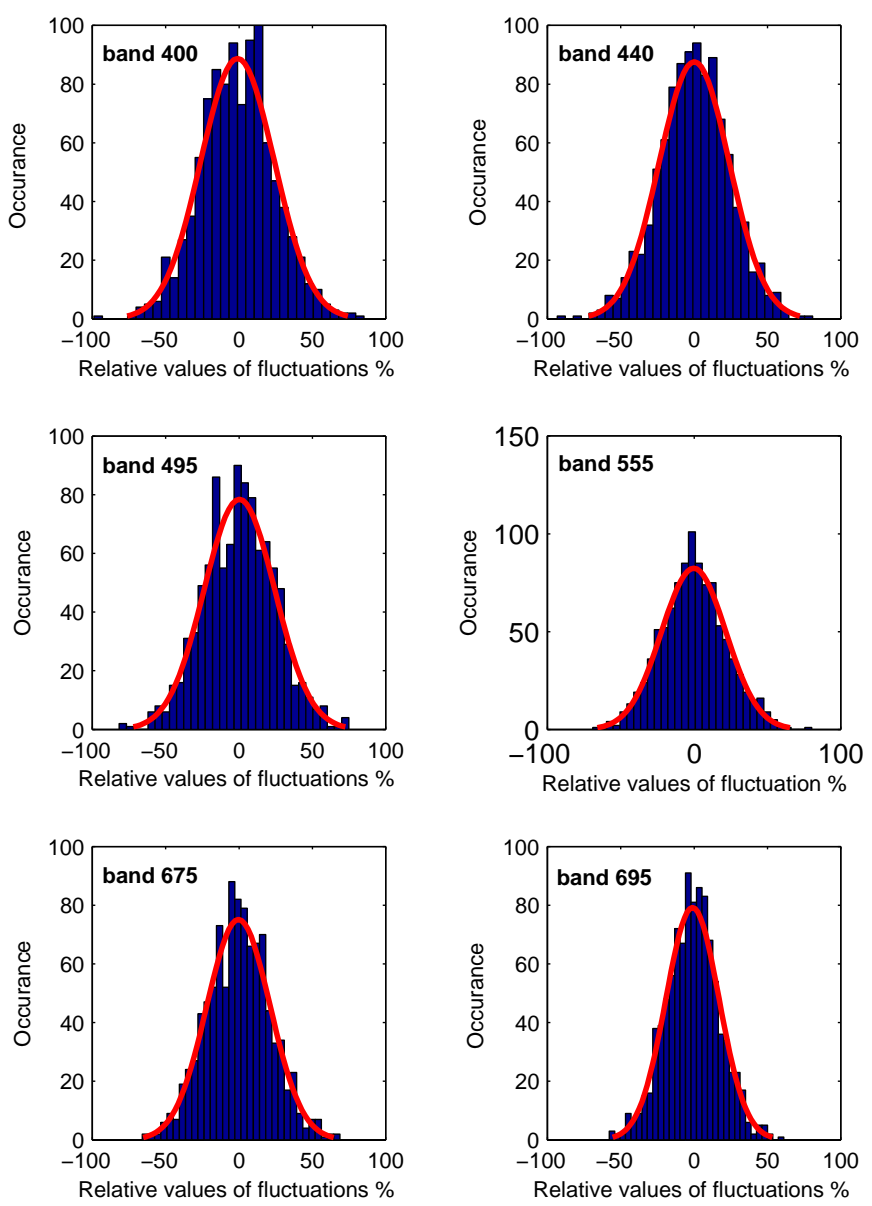

Figure 4. RMS-RE values in estimated $\sigma$ as function of wavelength. The values of $\sigma$ were derived from fluctuated mean and two field spectra corresponding to 0.05 and 0.95 quantiles.

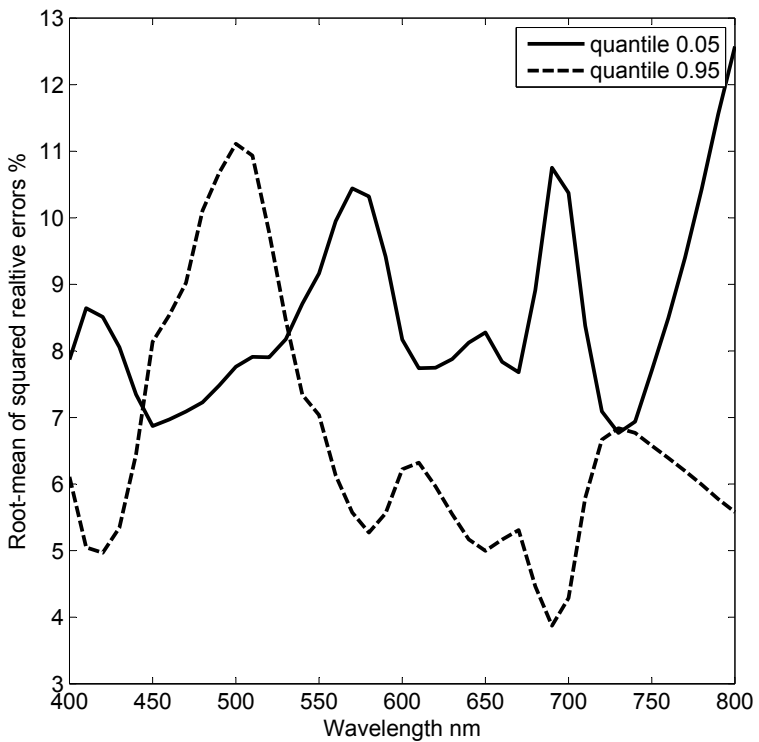


Figure 5. (a) Remote sensing reflectance of MERIS full resolution (FR) band 6 centered at $620 \mathrm{~nm}$; (b) derived standard deviation of the reduced resolution (RR) MERIS image using the FR pixels; (c-h) relative errors of derived (log) standard deviation values of RR-MERIS corresponding to quantiles $0.05,0.25,0.5,0.75,0.9$ and 0.95 .

(a): FR-MERIS Rs (620) [sr $\left.{ }^{-1}\right]$

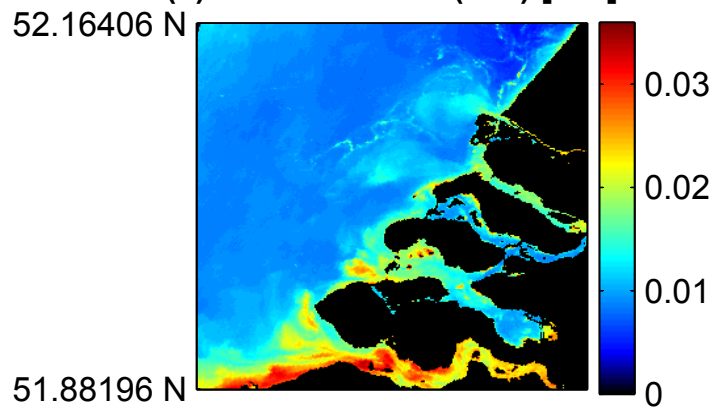

(c): errors in $\sigma(0.05$ quantile) [\%]

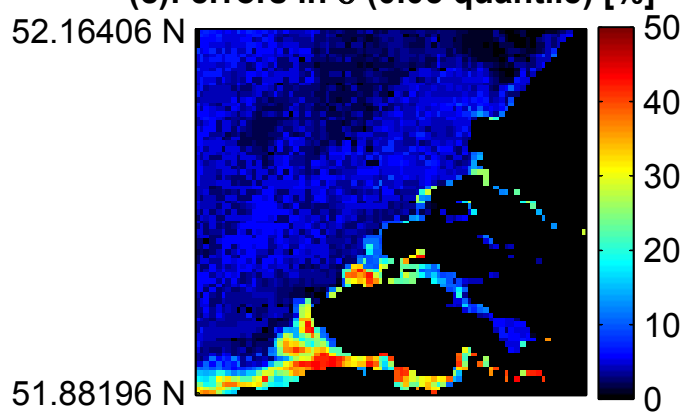

(e): errors in $\sigma$ (0.5 quantile) [\%]

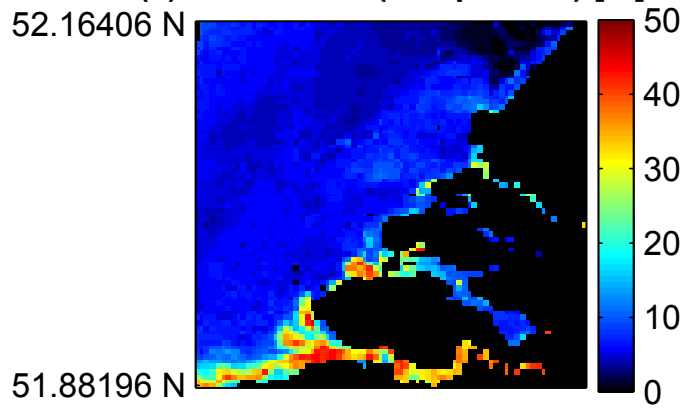

(g): errors in $\sigma$ (0.9 quantile) [\%]

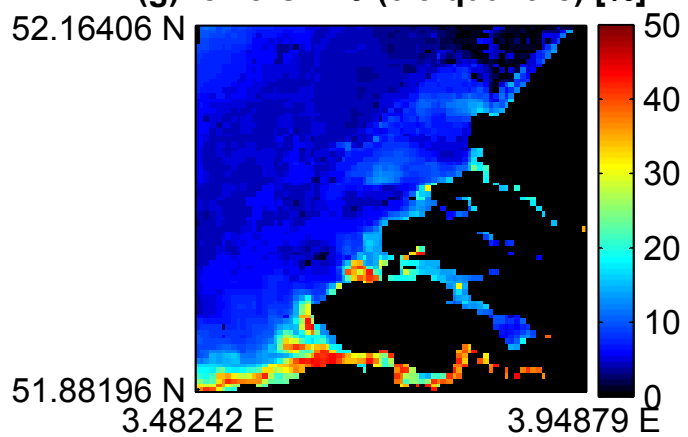

(b): $100 \times \sigma$ of RR-MERIS [sr ${ }^{-1}$ ]

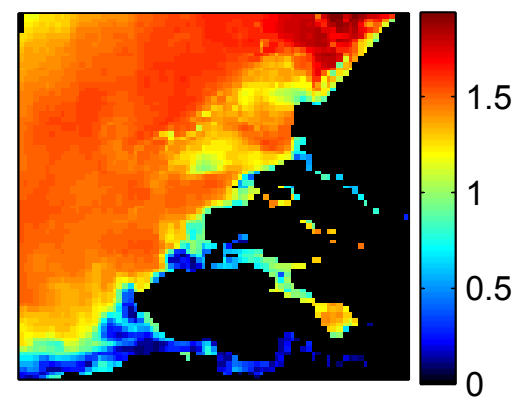

(d): errors in $\sigma(0.25$ quantile) [\%]

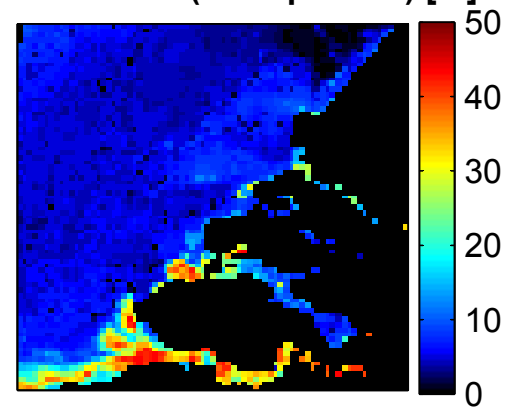

(f): errors in $\sigma$ (0.75 quantile) [\%]

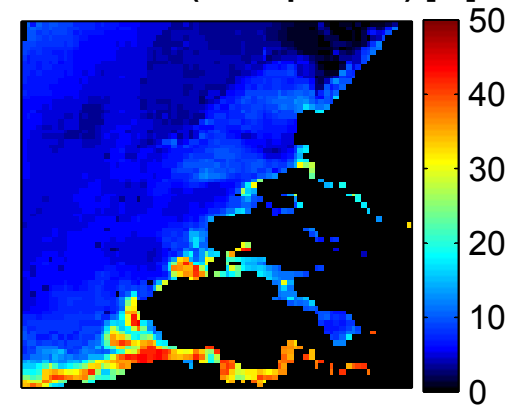

(h): errors in $\sigma$ (0.95 quantile) [\%]

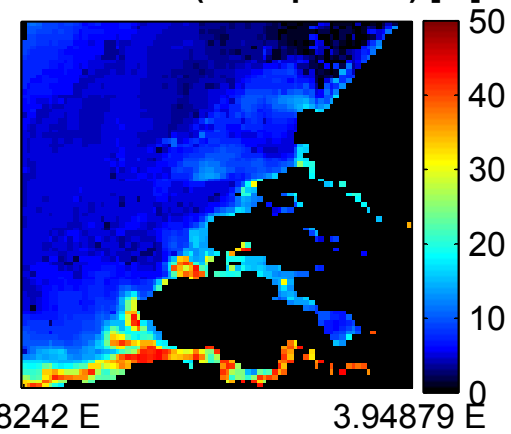




\section{Discussion}

Our method is formulated based on two steps. First we estimate the plausible range of IOPs. Second, we derive the posterior PDF of IOPs. In the first step we use the method of Bates and Watts [15] to construct a bound-like around field and aerospace spectra. These spectra are inverted to derive the plausible range of IOPs. In the second step, we use the cross-entropy (3) as a utility function. The cross-entropy in (3) can be rewritten as: joint entropy minus the posterior's entropy. Therefore maximizing (3) means maximizing the joint entropy between prior and posterior and minimizing the uncertainty about the posterior [18], resulting in an optimum posterior and maximum joint entropy. Equation (1) is then applied using the posterior PDF of IOPs to derive the empirical PDF of reflectance.

Validation with IOCCG data set shows that the root-mean of squared relative error is less than $15 \%$ for all possible field measurements. Moreover, derived values of variability are linearly related to the known values on a $\log$ scale with $\mathrm{R}^{2}>0.88$. Derived variability values from the green band, centered at $\sim 495 \mathrm{~nm}$, are more probable and are invariant to the position of in-situ measurements in the theoretical PDF. The stability of the model is tested by imposing random fluctuations to the observed aerospace mean. The retrieved spatial variabilities from fluctuated data are within $\pm 15 \%$ of the known values, with derived values from the 0.95 quantile being more accurate than those derived from the 0.05 quantile.

The proposed model is further tested with full and reduced resolution MERIS products covering part of the Dutch coastal waters. The highest errors in derived values of sub-pixels variability are in spatially homogenous areas. In these areas all quantile values are close to the mean and thus little information can be derived. This can also be observed in Figure 2. The results are slightly similar with respect to the different quantiles. However, derived variability from the 0.05 quantile was overestimated in turbid areas and provided good estimates in clear areas. The opposite was true for derived values using the 0.95 quantile, i.e., better results were obtained in turbid waters. This is logic because in turbid waters it is difficult to find clear water pixels and in clear waters is difficult to find turbid water pixels. However, on the borders of the turbidity zone the method worked quite well. These areas exhibit the highest spatial variability at a given time. Our method derived accurate variability estimates in these edge-like areas. This behavior is apparent in Figure 5. Note the diagonal stripe from South-West to North-East separating the Dutch coastal waters and the high reflectance area in the North-West region of the image. This stripe indicates low error in derived variability.

Since atmospheric correction is a significant issue in water remote sensing [25], imperfect atmospheric correction can lead to a significant error if not properly treated. A proper treatment is to combine our Bayesian model with the method of Salama and Stein [9] to decompose the difference between satellite and field observations into error and scale components. The error component can be treated as in Salama and Stein [9] and the scale component can be quantified using our Bayesian model.

\section{Conclusions}

In this paper we developed and applied a Bayesian approach to address the scale variability between point and aerospace measurements above water. The model used the differences between the field and aerospace observed spectra to derive prior information on the IOPs. We then applied Bayesian inference to derive the optimum posterior distribution of IOPs by maximizing the joint entropy of the 
prior-posterior. Our approach provided information about the sub-scale variability of match-up pixel on the IOPs and radiometric levels. We, further, showed that match-up sites for radiometric quantity could be inhomogeneous and preferably located on the edge of the turbidity zone. Information on the sub-scale variability of geo-biophysical processes will facilitate planning of calibration and validation of future sensors, resolving the critical scale of variability of an observed feature and improving the assimilation of EO products into model grid and field data. Although the approach was developed for radiometric quantities in a match-up pixel, it has the potential to be applied on bio-geophysical properties using prior knowledge on their plausible ranges. In addition, we believe that our methodology is general and applicable to land surface studies. The same principle applies: utilizing prior knowledge about geo-biophysical quantities to derive sub-scale variability of satellite pixel. However, the proposed model needs a more extensive validation with different data sets on land parameters.

\section{Acknowledgment}

The authors would like to thank the European Space Agency (ESA) for supplying MERIS data, Wim Timmermans from ITC, the Netherlands for providing technical assistance related to the Eagle 2006 data set, the Management Unit of the North Sea Mathematical Models (MUMM, Belgium) for maintaining the Oostende site. Anonymous reviewers are acknowledged for their comments.

\section{References}

1. Harding, L., Jr.; Magnusona, A.; Malloneea, M. SeaWiFS retrievals of chlorophyll in Chesapeake Bay and the mid-Atlantic bight. Estuar. Coast. Shelf Sci. 2005, 62, 75-94.

2. Bailey, S.; Werdell, J. A multi-sensor approach for the on-orbit validation of ocean color satellite data products. Remote Sens. Environ. 2006, 102, 12-23.

3. McClain, C.; Feldman, G.; Hooker, S. An overview of the SeaWiFS project and strategies for producing a climate research quality global ocean bio-optical time series. Deep-Sea Res. Pt. II-Top. St. Oce. 2004, 51, 5-42.

4. Garcia, C.; Garcia, V.; McClain, C. Evaluation of SeaWiFS chlorophyll algorithms in the Southwestern Atlantic and Southern Oceans. Remote Sens. Environ. 2005, 95, 125-137.

5. Mélin, F.; Berthon, J.; Zibordi, G. Assessment of apparent and inherent optical properties derived from SeaWiFS with field data. Remote Sens. Environ. 2005, 97, 540-553.

6. Darecki, M.; Stramski, D. An evaluation of MODIS and SeaWiFS bio-optical algorithms in the Baltic Sea. Remote Sens. Environ. 2004, 89, 326-350.

7. Chang, G.; Gould, R. Comparisons of optical properties of the coastal ocean derived from satellite ocean color and in situ measurements. Opt. Express 2006, 14, 10149-10163.

8. Hyde, K.; O’Reilly, J.; Oviatt, C. Validation of SeaWiFS chlorophyll-a in Massachusetts Bay. Cont. Shelf Res. 2007, 27, 1677-1691.

9. Salama, M.S.; Stein, A. Error decomposition and estimation of inherent optical properties. Appl. Opt. 2009, 48, 4926-4962. 
10. IOCCG Remote Sensing of Inherent Optical Properties: Fundamentals, Tests of Algorithms, and Applications. Lee, Z.P., Ed.; IOCCG Report Number 5; International Ocean-Colour Coordinating Group: Dartmouth, Canada, 2006.

11. Gordon, H.; Brown, O.; Evans, R.; Brown, J.; Smith, R.; Baker, K.; Clark, D. A semianalytical radiance model of ocean color. J. Geophys. Res. 1988, 93, 10,909-10,924.

12. Salama, M.S.; Dekker, A.G.; Su, Z.; Mannaerts, C.M.; Verhoef, W. Deriving inherent optical properties and associated inversion-uncertainties in the Dutch lakes. Hydrol. Earth Syst. Sci. 2009, 13, 1113-1121.

13. Salama, M.S.; Shen, F. Stochastic inversion of ocean color data using the cross-entropy method. Opt. Express 2010, 18, 479-499.

14. Savelieva, E.; Demyanov, V.; Maignan, M. Geostatistics: Spatial predictions and simulations. In Adanced Mapping of Environmental Data: Geostatistics, Machine Learning and Bayesian Maximum Entropy; Kanevski, M., Ed.; Willey: Hoboken, NJ, USA, 2008; pp. 47-94.

15. Bates, D.; Watts, D. Nonlinear Regression Analysis and Its Applications; John Wiley Sons, Inc.: Hoboken, NJ, USA, 1988.

16. Campbell, J. The log-normal distribution as a model for bio-optical variability in the sea. $J$. Geophys. Res. 1995, 100, 13,237-13,254.

17. Rubinstein, R.; Kroese, D. The Cross-Entropy Method: A Unified Approach to Combinatorial Optimization, Monte-Carlo Simulation, and Machine Learning; Springer: New York, NY, USA, 2004.

18. Wu, N. The Maximum Entropy Method (Springer Series in Information Sciences); Springer: Berlin, Germany, 1997; Volume 32.

19. Sydor, M.; Gould, R.; Arnone, R.; Haltrin, V.; Goode, W. Uniqueness in remote sensing of the inherent optical properties of ocean water. Appl. Opt. 2004, 43, 2156-2162.

20. Ansari, A.; Bradley, R. Rank-Sum tests for dispersions. Ann. Math. Statist. 1960, 31, 1174-1189.

21. Laws, E. Mathematical Methods for Oceanographers: An introduction; John Wiley Sons, Inc.: New York, NY, USA, 1997.

22. Su, Z.; Timmermans, W.; van der Tol, C.; Dost, R.; Bianchi, R.; Gomez, J.A.; House, A.; Hajnsek, I.; Menenti, M.; Magliulo, V.; Esposito, M.; Haarbrink, R.; Bosveld, F.; Rothe, R.; Baltink, H.; Vekerdy, Z.; Sobrino, J.; Timmermans, J.; van Laake, P.; Salama, M.S.; van der Kwast, H.; Claassen, E.; Stolk, A.; Jia, L.; Moors, E.; O., H.; Gillespie, A. EAGLE 2006-Multi-purpose, multi-angle and multi-sensor in-situ and airborne campaigns over grassland and forest. Hydrol. Earth Syst. Sci. 2009, 13, 833-845.

23. Vermote, E.; Tanre, D.; Deuze, J.; Herman, M.; Morcrette, J. Second simulation of the satellite signal in the solar spectrum, 6S: An overview. IEEE Trans. Geosci. Remote Sens. 1997, 35, 675-686.

24. Salama, M.S.; Shen, F. Simultaneous atmospheric correction and quantification of suspended particulate matters from orbital and geostationary earth observation sensors. Estuar. Coast. Shelf Sci. 2010, 86, 499-511.

25. Salama, S.; Monbaliu , J.; Coppin, P. Atmospheric correction of advanced very high resolution radiometer imagery. Int. J. Remote Sens. 2004, 25, 1349-1355. 
26. Pope, R.; Fry, E. Absorption spectrum (380-700nm) of pure water: II, Integrating cavity measurements. Appl. Opt. 1997, 36, 8710-8723.

27. Mobley, C. Light and Water Radiative Transfer in Natural Waters; Academic Press: London, UK, 1994.

28. Lee, Z.; Carder, K.; Mobley, C.; Steward, R.; Patch, J. Hyperspectral remote sensing for shallow waters: 2. Deriving bottom depths and water properties by optimization. Appl. Opt. 1999, 38, 3831-3843.

29. Maritorena, S.; Siegel, D.; Peterson, A. Optimization of a semianalytical ocean color model for global-scale applications. Appl. Opt. 2002, 41, 2705-2714.

30. Bricaud, A.; Morel, A.; Prieur, L. Absorption by dissolved organic-matter of the sea (yellow substance) in the UV and visible domains. Limnol. Oceanog. 1981, 26, 43-53.

31. Kopelevich, O. Small-parameter model of optical properties of sea waters. In Ocean Optics; Monin, A., Ed.; Nauka: Moscow, Russia, 1983; Volume 1, pp. 208-234.

32. Petzold, T. Volume scattering functions for selected ocean waters. In Light in the Sea; Tyler, J., Ed.; Dowden, Hutchinson and Ross: Stroudsburg, PA, USA, 1977; Volume 12, pp. 150-174.

\section{Appendix}

\section{Parametrization of IOPs}

The absorption and scattering coefficients of water molecules, $a_{w}$ and $b_{w}$, were assumed constants and obtained from [26,27], respectively. The total absorption of phytoplankton pigments $a_{p h y}$ is approximated as [28]:

$$
a_{p h y}(\lambda)=a_{0}(\lambda) a_{p h y}(0.44)+a_{1}(\lambda) a_{p h y}(0.44) \ln a_{p h y}(0.44)
$$

where $a_{0}(\lambda)$ and $a_{1}(\lambda)$ are empirical coefficients. The absorption effects of detritus and dissolved organic matter are combined due to the similar spectral signature [29] and approximated using the model [30]:

$$
a_{d g}(\lambda)=a_{d g}(440) \exp [-s(\lambda-440)]
$$

where $s$ is the spectral exponent with value $\sim 0.021 \mathrm{~nm}^{-1}$. The scattering coefficient of SPM $b_{\text {spm }}$ is parameterized as [31]:

$$
b_{\text {spm }}(\lambda)=b_{\text {spm }}(550)\left(\frac{550}{\lambda}\right)^{y}
$$

where $y$ is the spectral shape parameter $\sim 1.7$. The backscattering fraction of SPM is estimated from the "San Diego harbor" scattering phase function of Petzold [32], i.e., $b_{b}=0.0182 \times b$.

\section{Estimating the gradient of radiometric observation}

Observed remote sensing reflectance can be approximated as being the sum of the model best-fit $R s_{m}(\lambda)$ and its deviations from the observed one $\epsilon(\lambda)$ :

$$
R s(\lambda)=R s_{m}(\lambda)+\epsilon(\lambda)
$$


The term $R s_{m}(\lambda)$ is obtained from fitting the model in (1) to the radiometric observation of ocean color or/and field sensors. The error $\epsilon(\lambda)$ is a lumped term that includes model goodness-of-fit, measurements and atmospheric noises. For simplicity this term is assumed to be nearly independent the derived IOPs. The derivative of (8), with respect to the derived values, can then be written as:

$$
\frac{\Delta R s(\lambda)}{\Delta \text { iop }}=\frac{\Delta R s_{m}(\lambda)}{\Delta \text { iop }}+\frac{\Delta \epsilon(\lambda)}{\Delta \text { iop }}
$$

By definition of the least square minimization that was used to derive model-best-fit $R s_{m}(\lambda)$, we have:

$$
\frac{\Delta \epsilon(\lambda)}{\Delta \text { iop }} \approx 0
$$

Equation (9) can then be reduced to:

$$
\frac{\Delta R s(\lambda)}{\Delta \text { iop }} \approx \frac{\Delta R s_{m}(\lambda)}{\Delta \text { iop }}
$$

The simplification in (11) implies that the gradient of measured remote sensing reflectance can be approximated by the gradient of the model in (1) which can easily be computed.

\section{Driving the standard deviation from the plausible range}

Salama and Stein [9] has developed a method to estimate the standard deviation of a log-normally distributed population from its plausible range. Their method starts by generating random values from a normal distribution with zero mean and unity standard deviation, $\mathrm{N}(0,1)$. The generated values of $\mathrm{N}(0,1)$ satisfy an imposed acceptance-rejection condition. This condition requires that the ratio (13) defines a unique ordered pair of $\alpha$ :

$$
\alpha_{u}=\frac{\text { iop }_{u}-\text { iop }_{o b s}}{\sigma}
$$

where $\operatorname{iop}_{u}$, iop $_{o b s}$ and $\sigma$ are the upper bound of the IOPs; the expectation (derived from model best-fit to observation) and the unknown standard deviation of the population, respectively. All IOPs are log-transformed first. For the lower bound of IOPs, $\operatorname{iop}_{l}$, one can establish the ratio:

$$
r_{u, l}=\frac{\alpha_{u}}{\alpha_{l}}=\frac{\operatorname{iop}_{u}-\operatorname{iop}_{o}}{\operatorname{iop}_{l}-\operatorname{iop}_{o}}
$$

Three look-up tables (LUTs) are then created from the generated $\mathrm{N}(0,1)$ values. These LUTs correspond to the following three scenarios:

$$
\begin{aligned}
& \operatorname{iop}_{o}>\operatorname{iop}_{u}>\operatorname{iop}_{l} \\
& \operatorname{iop}_{o}<\operatorname{iop}_{l}<\operatorname{iop}_{u} \\
& \operatorname{iop}_{l}<\operatorname{iop}_{o}<\operatorname{iop}_{u}
\end{aligned}
$$

The ratio in (13) is first estimated from the log-transformed IOPs values. Based on the value of this ratio a lookup table is selected and searched to find the best-fit. One of the corresponding pair is then used in (12) to compute the standard deviation of the prior PDF. 


\section{Added random noise}

Random normal fluctuations are assumed to be wavelengths dependent with zero mean and standard deviation calculated from the theoretical PDF, see Figure 3.

(c) 2010 by the authors; licensee MDPI, Basel, Switzerland. This article is an Open Access article distributed under the terms and conditions of the Creative Commons Attribution license (http://creativecommons.org/licenses/by/3.0/.) 